\title{
Recurrent gain-of-function USP8 mutations in Cushing's disease
}

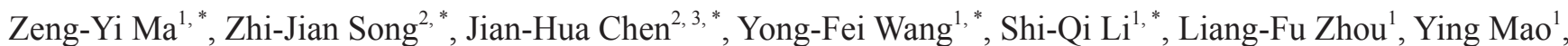
Yi-Ming $\mathrm{Li}^{4}$, Rong-Gui $\mathrm{Hu}^{5}$, Zhao-Yun Zhang ${ }^{4}$, Hong-Ying Ye ${ }^{4}$, Ming Shen ${ }^{1}$, Xue-Fei Shou ${ }^{1}$, Zhi-Qiang Li ${ }^{2}$, Hong Peng, ${ }^{5}$, Qing-Zhong Wang ${ }^{2}$, Dai-Zhan Zhou ${ }^{2}$, Xiao-Lan Qin ${ }^{2}$, Jue Ji ${ }^{2}$, Jie Zheng ${ }^{2}$, Hong Chen ${ }^{6}$, Yin Wang ${ }^{6}$, Dao-Ying Geng ${ }^{7}$, Wei-Jun Tang ${ }^{7}$, Chao-Wei Fu ${ }^{8}$, Zhi-Feng Shi ${ }^{1}$, Yi-Chao Zhang ${ }^{1}$, Zhao Ye ${ }^{1}$, Wen-Qiang He ${ }^{1}$, Qi-Lin Zhang ${ }^{1}$, Qi-Sheng Tang ${ }^{1}$, Rong Xie ${ }^{1}$, Jia-Wei Shen ${ }^{2}$, Zu-Jia Wen ${ }^{2}$, Juan Zhou ${ }^{2}$, Tao Wang ${ }^{9}$, Shan Huang ${ }^{9}$, Hui-Jia Qiu ${ }^{1}$, Ni-Dan Qiao ${ }^{1}$, Yi Zhang ${ }^{1}$, Li Pan ${ }^{1}$, Wei-Min Bao ${ }^{1}$, Ying-Chao Liu ${ }^{10}$, Chuan-Xin Huang ${ }^{11}$, Yong-Yong $\mathrm{Shi}^{2,12}$, Yao Zhao

${ }^{I}$ Department of Neurosurgery, Shanghai Pituitary Tumor Center, Huashan Hospital, Shanghai Medical College, Fudan University, Shanghai 200040, China; ${ }^{2}$ Bio-X Institutes, Ministry of Education Key Laboratory for the Genetics of Developmental and Neuropsychiatric Disorders, Institute of Social Cognitive and Behavioral Sciences, Shanghai Jiao Tong University, Shanghai 200030, China, ${ }^{3}$ Shanghai Key Laboratory of Psychotic Disorders, Shanghai Mental Health Center, Shanghai Jiao Tong University School of Medicine, Shanghai 200030, China, ${ }^{4}$ Department of Endocrinology, Huashan Hospital, Shanghai Medical College, Fudan University, Shanghai 200040, China, ${ }^{5}$ State Key Laboratory of Molecular Biology, Institute of Biochemistry and Cell Biology, Shanghai Institutes for Biological Sciences, Chinese Academy of Sciences, Shanghai 200031. China; ${ }^{6}$ Department of Neuropathology, Huashan Hospital, Shanghai Medical College, Fudan University, Shanghai 200040, China; ${ }^{7}$ Department of Radiology, Huashan Hospital, Shanghai Medical College, Fudan University, Shanghai 200040, China; ${ }^{8}$ Department of Epidemiology, School of Public Health, Fudan University, Shanghai 200032, China; ${ }^{9}$ Department of Neurosurgery, Shanghai 5th People's Hospital, Shanghai 200240, China; ${ }^{10}$ Department of Neurosurgery, Provincial hospital, Shandong University, Jinan, Shandong 250021, China; ${ }^{11}$ Shanghai Institute of Immunology \& Department of Immunobiology and Microbiology, Shanghai Jiao Tong University School of Medicine, Shanghai 200025, China, ${ }^{12}$ Shandong Provincial Key Laboratory of Metabolic Disease, the Affiliated Hospital of Qingdao University, Qingdao, Shandong 266003, China

Cushing's disease, also known as adrenocorticotropic hormone (ACTH)-secreting pituitary adenomas (PAs) that cause excess cortisol production, accounts for up to $85 \%$ of corticotrophin-dependent Cushing's syndrome cases. However, the genetic alterations in this disease are unclear. Here, we performed whole-exome sequencing of DNA derived from 12 ACTH-secreting PAs and matched blood samples, which revealed three types of somatic mutations in a candidate gene, USP8 (encoding ubiquitin-specific protease 8), exclusively in exon 14 in 8 of 12 ACTH-secreting PAs. We further evaluated somatic USP8 mutations in additional 258 PAs by Sanger sequencing. Targeted sequencing further identified a total of 17 types of USP8 variants in 67 of 108 ACTH-secreting PAs (62.04\%). However, none of these mutations was detected in other types of PAs $(n=150)$. These mutations aggregate within the 14-3-3 binding motif of USP8 and disrupt the interaction between USP8 and 14-3-3 protein, resulting in an elevated capacity to protect EGFR from lysosomal degradation. Accordingly, PAs with mutated USP8 display a higher incidence of EGFR expression, elevated EGFR protein abundance and mRNA expression levels of $P O M C$, which encodes the precursor of ACTH. PAs with mutated USP8 are significantly smaller in size and have higher ACTH production than wildtype PAs. In surgically resected primary USP8-mutated tumor cells, USP8 knockdown or blocking EGFR effectively attenuates ACTH secretion. Taken together, somatic gain-of-function USP8 mutations are common and contribute to ACTH overproduction in Cushing's disease. Inhibition of USP8 or EGFR is promising for treating USP8-mutated corticotrophin adenoma. Our study highlights the potentially functional mutated gene in Cushing's disease and provides insights into the therapeutics of this disease.

Keywords: Cushing's disease; pituitary adenomas; USP8; mutation; whole-exome sequencing Cell Research (2015) 25:306-317. doi:10.1038/cr.2015.20; published online 13 February 2015 


\section{Introduction}

Adrenocorticotropic hormone (ACTH)-secreting pituitary adenomas (PAs), referred to as Cushing's disease, arise from pituitary corticotroph cells and secrete large amounts of ACTH, promoting the adrenal glands to produce elevated levels of cortisol and then induce endogenous hypercortisolism $[1,2]$. Cushing's disease accounts for up to $85 \%$ of corticotrophin-dependent Cushing's syndrome cases [3]. Cushing's disease is a severe and potentially fatal disease. Patients may have many clinical features such as central obesity, hypertension, cardiovascular disease and metabolic syndrome [2, 4], which are variably present in any given case. Both the diagnosis of hypercortisolism and the preoperative location of PAs in Cushing's disease are complicated and sometimes difficult $[1,4]$. Currently, the first-line treatment of Cushing's disease is pituitary adenomectomy, generally performed via a transsphenoidal approach. However, only some patients achieve initial remission after surgery and PAs tend to recur after periods of remission [5], emphasizing the need to develop novel therapeutic approaches for Cushing's disease.

ACTH hypersecretion is a hallmark of Cushing's disease and reported to be caused by several molecular defects. For example, downregulation of Brg1 and histone deacetylase 2 (HADC2) is found in Cushing's disease, which promotes proopiomelanocortin $(P O M C)$ transcription [6]. Reduced expression of the cell cycle inhibitor p27 (Kip1) is detected in human Cushing's adenoma [7], and mice with disrupted p27(Kip1) develop ACTH-secreting PAs [8]. High expression of EGFR is frequently observed in ACTH-secreting PAs and blocking EGFR signaling effectively inhibits ACTH secretion [9, 10]. However, the genetic basis for these molecular alterations remains elusive.

Several genetic alterations have been described in PAs. For example, somatic activating mutations of guanine nucleotide-binding protein $\alpha$ subunit $(G N A S)$ are found in $30 \%-40 \%$ of sporadic somatotroph adenomas and account for McCune-Albright syndrome accompanied by PAs $[11,12]$. Germline AIP mutations are found in sporadic PA patients without a family history of this disease $[13,14]$. However, ACTH-secreting PAs are rarely reported to associate with adenoma-predisposing syndromes as well as mutations of GNAS or AIP [15]. Although case reports of mutations in $p 53$ [16], DAX-1
[17], DICER1 [18] or the glucocorticoid receptor gene NR3C1 [19] have been described in ACTH-secreting PAs or pituitary blastomas, the prevalent genetic alterations in these adenomas are still unknown.

In this study, we identified a total of 17 types of USP 8 (ubiquitin-specific protease 8) variants, which occurred in more than $60 \%$ of $\mathrm{ACTH}$-secreting corticotrophin adenomas. These mutations could induce ACTH overproduction via deregulation of EGFR signaling and may lead to different clinical phenotypes. We further showed that USP8 knockdown or gefitinib (a clinically available EGFR inhibitor) treatment significantly reduced ACTH secretion in primary USP8-mutated corticotrophin adenoma cells, but not in wild-type cells.

\section{Results}

\section{Somatic USP8 mutations in Cushing's disease}

To search for the genetic alterations in ACTH-secreting PAs, whole-exome sequencing was performed in DNA from 12 tumors and matched blood samples (Supplementary information, Table S1). Mean tumor purity was estimated as $90 \%$. The average sequencing depth was $74 \times(42 \times$ to $162 \times)$ for the tumors and $113 \times(47 \times$ to $175 \times$ ) for blood (Supplementary information, Figure S1A). Furthermore, 94\% (91\%-95\%) of target regions were covered by at least $10 \times$ sequencing depth (Supplementary information, Figure S1B). This study revealed a low number of somatic mutations (median, 5; range, 1-9) per case and a total of 45 non-synonymous and 12 synonymous somatic mutations (Figure 1A and Supplementary information, Table S2), which were further validated by Sanger sequencing.

Except for nonsense mutation of $N R 3 C 1$ in one patient, no mutations were detected in any previously reported genes associated with adrenal Cushing's syndrome or PAs, including GNAS or PRKACA (Figure 1A). Strikingly, three recurrent somatic mutations (c.CTC2151$2153 \mathrm{del} / \mathrm{p} . \mathrm{S} 718 \mathrm{del}$; c.C2159G/p.P720R; c.T2152C/ p.S718P), exclusively in exon 14 of the USP8 gene, were found in 8 out of 12 tumors (Figure 1A). Sanger sequencing revealed that there was no allelic imbalances of USP8 mutations in tumor tissues (Supplementary information, Figure S2), indicating a heterozygous status of these mutations, which is consistent with the observation by exome sequencing that their variant allele frequencies were comparable to those of other somatic mutations in

${ }^{\mathrm{c}}$ Tel/Fax: +86-21-52888683; E-mail: huangcx@shsmu.edu.cn Received 28 January 2015; revised 30 January 2015; accepted 2 February 2015; published online 13 February 2015

\footnotetext{
*These five authors contributed equally to this work. Correspondence: Yong-Yong Shi ${ }^{\mathrm{a}}$, Yao Zhao ${ }^{\mathrm{b}}$, Chuan-Xin Huang ${ }^{\mathrm{c}}$ ${ }^{a}$ Tel/Fax: +86-21-62932151; E-mail: shiyongyong@gmail.com

${ }^{\mathrm{b}} \mathrm{Tel} / \mathrm{Fax}$ : +86-21-52888728; E-mail: zhaoyaohs@vip.sina.com
} 
A

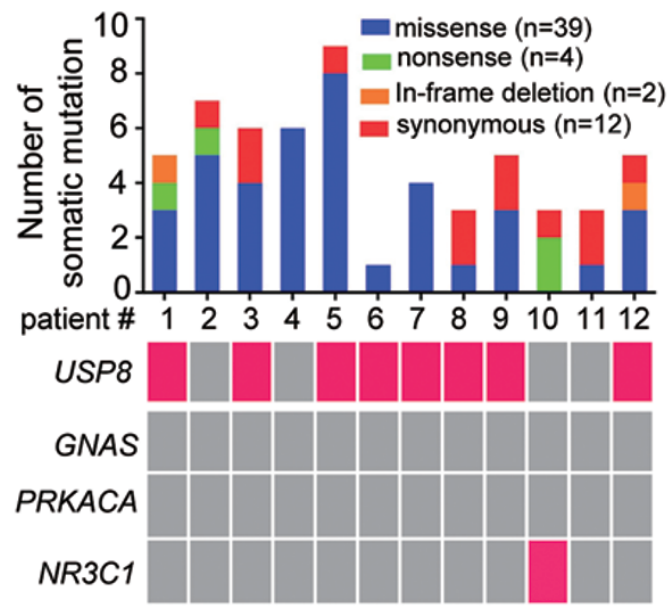

B

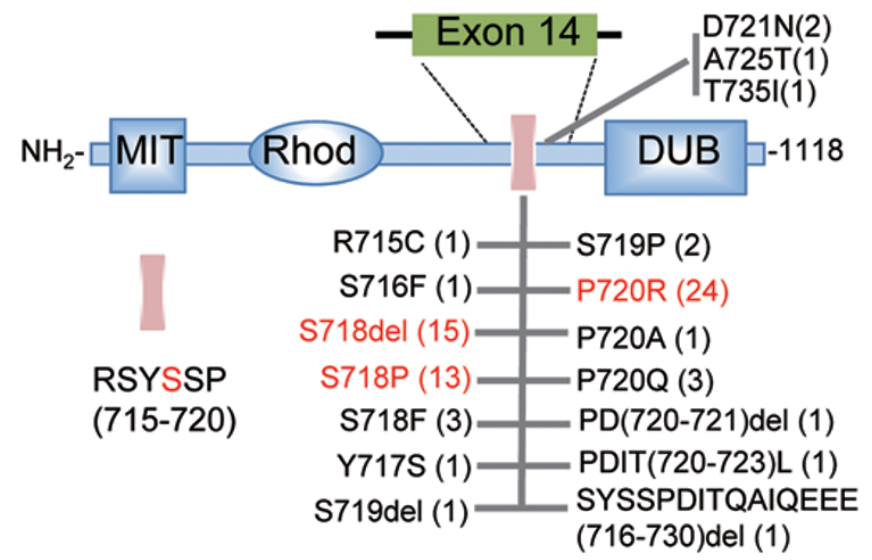

Figure 1 Recurrent USP8 mutations in Cushing's disease. (A) The number of somatic mutations (top) and the mutational status of the indicated gene (bottom) in each patient, as revealed by whole-exome sequencing and Sanger sequencing. The total number of each type of somatic mutation in 12 cases is shown on the top right. Patients carrying the indicated mutated gene are marked in pink (bottom). (B) Schematic diagram of USP8 domains and landscape of USP8 alterations in the 14-3-3 binding motif and its nearby region. These alterations were identified in 108 patients with Cushing's disease by Sanger sequencing. The number of cases with the indicated USP8 mutation among 108 patients is denoted in parentheses. The purple bar represents the 14-3-3 binding motif of USP8, and its amino acid sequence is shown on the bottom left. MIT, microtubule interaction and transport domain; Rhod, rhodanese-like domain; DUB, deubiquitinating domain.

tumor cells (Supplementary information, Figure S3). Except for USP8 mutations, no other mutations occurred in more than one tumor tissue (Supplementary information, Table S2). Finally, no copy number variation (CNV) of the USP8 gene was detected in $12 \mathrm{ACTH}$-secreting PAs based on whole-exome sequencing data.

To study the prevalence of USP 8 mutations in various PAs, we screened an additional 258 PAs including 108 ACTH-secreting, 50 growth hormone (GH)-secreting, 50 prolactin (PRL)-secreting and 50 non-functioning (NF) PAs (Supplementary information, Table S1). In addition to the three types of USP 8 mutations detected above, targeted sequencing identified other 14 types of somatic USP8 mutations distributed in 67 out of 108 ACTH-secreting PAs (62.04\%, Figure 1B and Supplementary information, Figure S4). Notably, the S718del, P720R and S718P mutations account for the vast majority of the USP8-mutated cases (77.61\%; Figure 1B). All these mutations were not detected in matched blood samples. All the 17 types of detected USP 8 mutations were not found in dbSNP v138 and 1000 Genomes Project. Except for c.C2159G/p.P720R and C2159A/p.P720Q mutations, other mutations were not found in the COSMIC v68 database, indicating that these mutations are novel somatic mutations. Strikingly, none of the other types of PAs had similar genetic alterations in exon 14 of USP8
(Supplementary information, Table S1). Taken together, these data indicate that USP8 mutations are common and unique genetic alterations in ACTH-secreting PAs.

\section{Functional characterization of USP8 mutants}

USP 8 is a deubiquitinating enzyme that protects growth factor receptors including epidermal growth factor receptor (EGFR) from degradation [20, 21]. EGFR activation promotes $P O M C$ transcription in primarily cultured human ACTH-secreting tumor cells [10]. USP8 contains a putative 14-3-3 binding motif RSXSXP that is encoded by part of exon 14 of USP8 in humans (Figure 1B) and is identically conserved in various species (Supplementary information, Figure S5). The phosphorylation of USP8 on the fourth serine (S718) of its 14-3-3 binding motif results in its binding with 14-3-3 proteins and catalytic inactivation $[22,23]$. A structural study demonstrated that within the 14-3-3 binding motif, the amino acid preferences in each position are very similar, and the phosphorylation of serine at the fourth position is essential for its binding ability [24]. Furthermore, this serine (S718) was most frequently disrupted via deletion or missense mutation in all USP8-mutated cases (37/75). Other affected amino acids were either in the 14-3-3 binding motif or its adjacent regions (Figure 1B). Thus, we examined whether three prevalent tumor-derived 
A

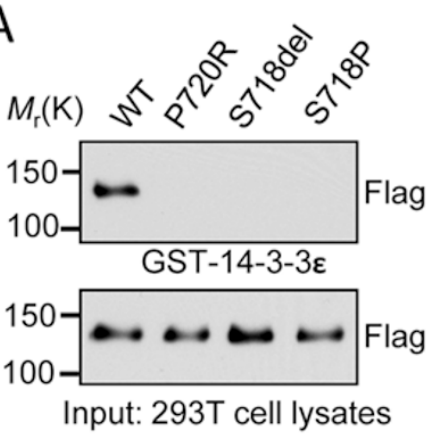

B

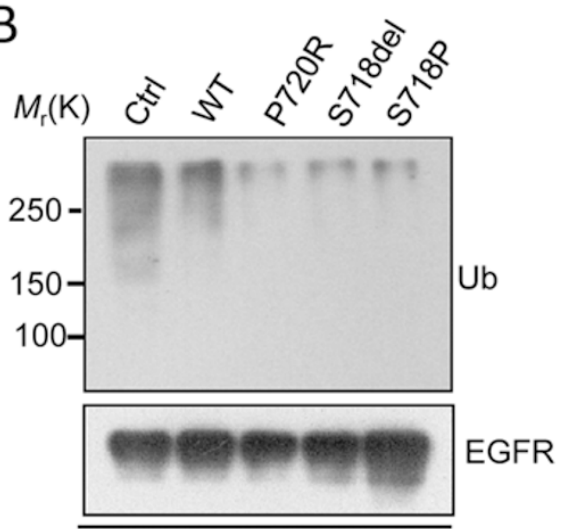

IP: EGFR

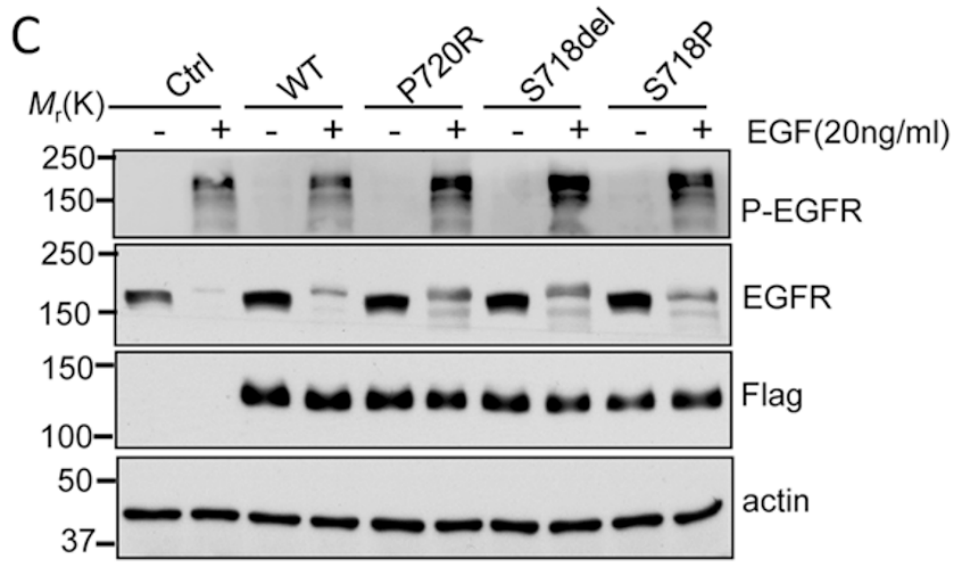

D

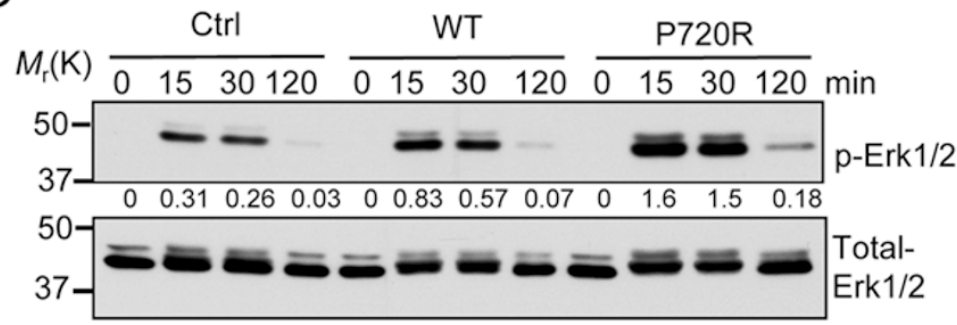

Figure 2 Molecular characterization of USP8 mutants. (A) USP8 mutants fail to bind 14-3-3 protein. Cell lysates from 293T cells expressing Flag-tagged WT-USP8 or the indicated USP8 mutants were incubated with GST-14-3-3ع immobilized on Glutathione-Sepharose beads. GST pull-down or input samples were immunoblotted with anti-Flag antibody. (B) Reduced EGFR ubiquitination in USP8 mutants. Cell lysates from HeLa cells stably expressing Flag-tagged WT-USP8, the indicated mutant or the vector only (Ctrl) were immunoprecipitated (IP) using anti-EGFR antibody, and the immunoprecipitation products were analyzed by immunoblotting with anti-EGFR and ubiquitin (Ub) antibodies. (C) Slower EGFR degradation in HeLa cells expressing USP8 mutant relative to WT. Serum-starved HeLa cells stably expressing Flag-tagged USP8 protein were treated with EGF $(20 \mathrm{ng} / \mathrm{ml})$ in the presence of cycloheximide $(25 \mu \mathrm{g} / \mathrm{ml})$ for $3 \mathrm{~h}$. Immunoblot analysis was performed to determine p-EGFR, EGFR and Flag-USP8 protein levels. (D) Enhanced EGF-induced Erk phosphorylation in USP8 P720R mutant. HeLa cells stably expressing WT, USP8 P720R mutants or the control were treated with EGF (20 ng/ml) for the indicated times. Cell lysates were subjected to immunoblot analysis for total Erk and phospho-Erk (p-Erk). The densitometric ratio of p-Erk1/2 to total-Erk1/2 is shown between panels. Three independent experiments were repeated with similar results.

USP8 mutations abolish or reduce USP8 binding ability with $14-3-3 \varepsilon$ and increase the capability of blocking EGFR degradation.

As expected, WT USP8 was shown to bind $14-3-3 \varepsilon$ in a GST pull-down assay. Interestingly, the three tested USP8 mutants were not detected in the $14-3-3 \varepsilon$ bound complexes, although they were expressed at levels similar to WT (Figure 2A). Moreover, EGFR ubiquitination was obviously reduced in cells transduced with mutant USP8 (Figure 2B). EGF treatment induced a faster degradation of EGFR protein in HeLa cells expressing WT USP 8 compared to mutants, although EGFR protein levels were comparable in these cells under serum-starved conditions (Figure 2C). Finally, EGF treatment induced a stronger phosphorylation of Erk $1 / 2$ in HeLa cells expressing mutant USP 8 (Figure 2D).

We next assessed the consequences of USP 8 mutation in tumor tissue. Immunochemical analysis demonstrated that the USP8 protein was ubiquitously expressed in normal pituitary tissue and in USP8 WT or mutated ACTH-secreting PAs at comparable levels (Figure 3A). However, only part of PAs were positive for EGFR staining, and EGFR-positive cases were more frequently detected in USP 8-mutated PAs $\left(P=0.01\right.$, Pearson's $\chi^{2}$ test), where they account for $\sim 80 \%$ of the cases $(28 / 35)$, compared to $50 \%$ in WT cases $(15 / 30)$. Notably, EGFR staining intensity in EGFR-positive tumors with WT USP 8 was generally lower (Figure 3A). Furthermore, 

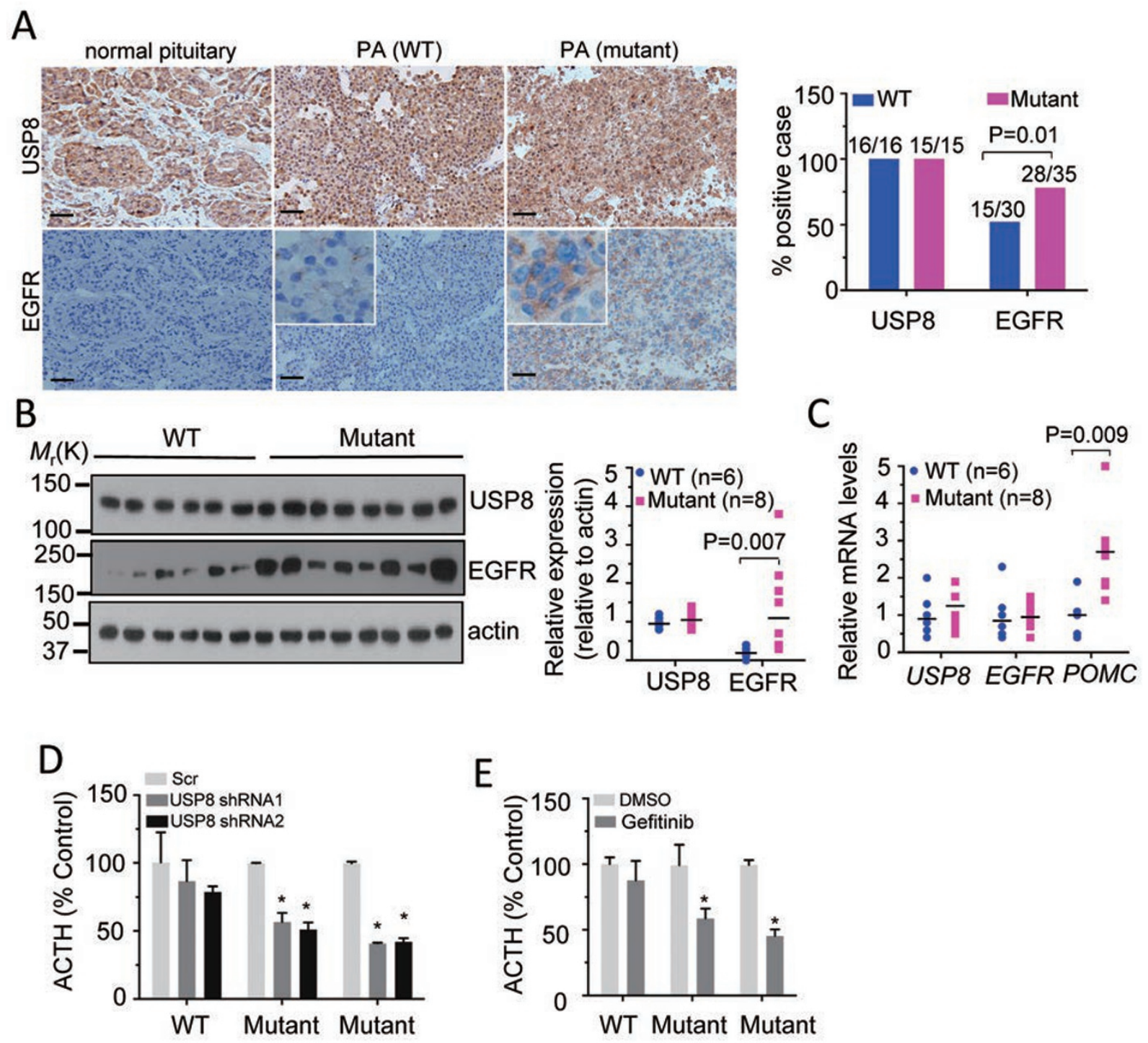

Figure 3 USP8 mutation enhances EGFR protein expression and POMC mRNA abundance in ACTH-secreting PAs. (A) Immunohistochemical staining for USP8 and EGFR in a representative normal pituitary, USP8 WT and mutated PAs. Scale bar, $50 \mu \mathrm{m}$. Inset, high magnification. In both WT and mutated groups, the percentage of USP8- and EGFR-positive cases was calculated (right). On top of the column, actual number of mutated samples over total analyzed. $P$ values were calculated by Pearson's $\chi^{2}$ test. (B) Immunoblot analysis of USP8, EGFR and actin levels in USP8 WT and mutated PAs (left). Each lane represents one case. The relative protein levels of USP8 and EGFR are normalized to actin and shown on the right. (C) Relative mRNA levels of USP8, EGFR and POMC in USP8 WT and mutated PAs, assessed by RT-qPCR analysis and normalized to the housekeeper gene HPRT. In B and C, a total of 6 WT and 8 USP8-mutated cases are analyzed and the number is indicated by $\mathrm{n}$. Each symbol represents an individual case and lines indicate the median. $P$ values were calculated by the Mann-Whitney U-test. (D) Suppression of ACTH secretion in primary human ACTH-secreting tumor cells after USP8 knockdown using lentivirus-mediated shRNA. (E) Decreased ACTH secretion in primary tumor cells treated with Gefitinib (1 $\mu \mathrm{M})$ for $48 \mathrm{~h}$. In D and $\mathbf{E}, \mathrm{ACTH}$ levels in culture media were measured with radioimmunoassy (RIA) and presented as \% of control media. Error bar indicates SEM of 3 replicates. ${ }^{*} P<0.05$ compared to control (Mann-Whitney $U$-test). One WT and two USP8-mutated PAs are shown.

immunoblot analysis demonstrated notably increased EGFR protein levels in half USP8-mutated PAs and Erk1/2 phosphorylation in most USP 8 -mutated PAs examined (Figure 3B and Supplementary information,
Figure S6). RT-qPCR analysis revealed a higher mRNA abundance of $P O M C(P=0.009$, Mann-Whitney $U$-test $)$, but not USP8 and EGFR, in ACTH-secreting PAs with USP8 mutations (Figure 3C). To assess the important 
role of USP8 mutation in ACTH production, we established surgically resected primary human ACTH-secreting tumor cells. USP8 knockdown resulted in a significant reduction of ACTH secretion in culture media from USP 8-mutated tumors cells $(P<0.05$, Mann-Whitney $U$-test), but not WT tumor cells (Figure 3D). Treatment of gefitinib, a clinically available EGFR inhibitor, also effectively attenuated ACTH secretion in USP8-mutated tumor cells (Figure 3E). Moreover, USP8 knockdown reduced EGFR protein level in USP8-mutated tumor cells (Supplementary information, Figure S7). Taken together, these results indicate that USP8 mutations contribute to the pathogenesis of ACTH-secreting PAs.

\section{Clinical phenotypes and USP8 mutational status}

Few genetic alterations have been linked to the clinical phenotype of Cushing's disease. We explored whether patients with Cushing's disease carrying USP8 mutations display distinct clinical features. USP 8 mutations were identified in both female and male patients, but their frequency was significantly higher in female patients $(67.7 \%)$ compared to male patients $(38.1 \%)(P=$ 0.01 , Pearson's $\chi^{2}$ test; Figure 4A). Contrast-enhanced T1-weighted magnetic resonance imaging (MRI) demonstrated that 16 of 39 USP8-WT PAs (41.0\%) were very large and extended into the parasellar space, including the cavernous sinus and the sphenoidal sinus. In contrast, the vast majority of USP8-mutated PAs was small in size (maximal diameter) and diffusely distributed within the sella, and only 8 of 63 cases (12.7\%) displayed parasellar invasion $\left(P=0.001\right.$, Pearson's $\chi^{2}$ test; Figure 4B and 4C) $[25,26]$. This morphological difference is further supported by the fact that USP8-mutated PAs were generally smaller in size compared to USP8-WT PAs $(P<0.001$,

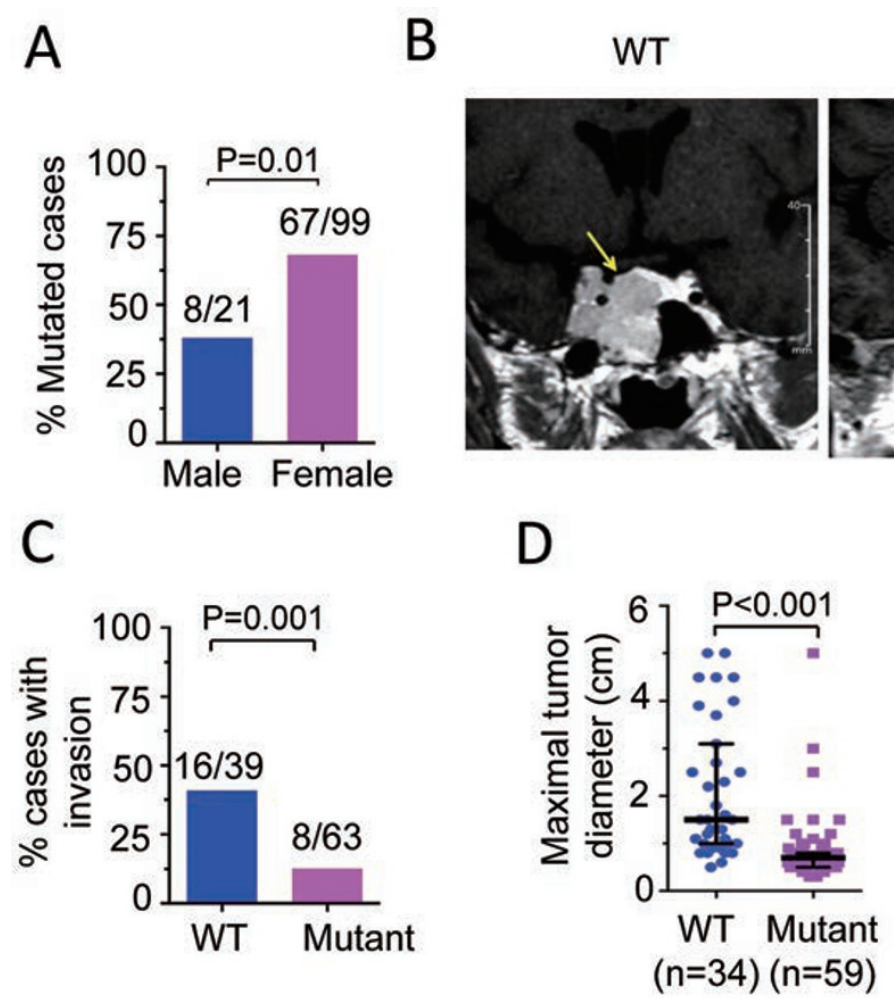

Figure 4 Clinical characteristics of patients with Cushing's disease in relation to USP8 mutational status. (A) Frequency of USP8 mutations in male and female patients with Cushing's disease. (B) Representative contrast-enhanced T1 weighted MRI of PAs with or without USP8 mutations. Tumor bulk is indicated by arrows. The WT tumor is very large and invasively extends into the sphenoidal sinus and the cavernous sinus, whereas the mutant tumor is markedly smaller in size and diffusely distributed within the sella. (C) Percentage of cases with invasion in both USP8 WT and mutated groups. Invasive adenomas were defined as fulfilling 1 of 2 conditions: (1) Hardy's modified classification grade III, IV and/or stage C, D and E; (2) Knosp classification grade III and IV. In A and C, the actual number of mutated cases over the total cases analyzed is shown on top of each column. $P$ values were calculated by Pearson's $\chi^{2}$ test. (D) Different maximal diameter between USP8 WT and mutated groups. Each symbol (dot) represents an individual case. Lines indicate the median with interquartile range (25\% and $75 \%)$. The number of patients with each genotype is indicated by $n$. $P$ values were calculated by the Mann-Whitney $U$-test. 
Table 1 A comparison of clinical characteristics of patients with ACTH-secreting PAs with or without USP8 mutations

\begin{tabular}{|c|c|c|c|}
\hline Characteristic & $\mathrm{WT}(n=45)$ & Mutant $(n=75)$ & $P$ value \\
\hline \multicolumn{4}{|l|}{ Age at diagnosis (Year) } \\
\hline Median & 40 & 36 & $0.08 \dagger$ \\
\hline \multicolumn{4}{|l|}{ Body Mass Index (BMI) } \\
\hline Median & 25.4 & 24.2 & $0.36 \dagger$ \\
\hline Median & 94.0 & 68.8 & \multirow[t]{2}{*}{$0.054 \uparrow$} \\
\hline Interquartile range & $60.6-163.0$ & $51.4-105.5$ & \\
\hline \multicolumn{4}{|l|}{ ACTH (pg/ml)/Size (cm) } \\
\hline Median & 51.0 & 103.0 & $<0.001 \dagger$ \\
\hline Interquartile range & $16.18-31.20$ & $18.35-29.41$ & $0.79 \dagger$ \\
\hline \multicolumn{4}{|l|}{ Urinary free cortisol $(\mu \mathrm{g} / 24 \mathrm{~h})$} \\
\hline Median & 650.24 & 552.64 & \multirow[t]{2}{*}{$0.39 \dagger$} \\
\hline Interquartile range & $412.13-1168.25$ & $262.75-977.47$ & \\
\hline \multicolumn{4}{|c|}{ Immediate postoperative biochemical remission ( $\%) \Phi$} \\
\hline Yes & $33(73.3)$ & $63(84.0)$ & \multirow[t]{2}{*}{$0.16 \ddagger$} \\
\hline No & $12(26.7)$ & $12(16.0)$ & \\
\hline \multicolumn{4}{|l|}{ Postoperative recurrence $(\%) Я$} \\
\hline Yes & $8(25.8)$ & $12(20.7)$ & $0.58+$ \\
\hline
\end{tabular}

†Values were compared by Mann-Whitney $U$-test.

tValues were compared by Pearson's $\chi^{2}$ test.

IImmediate postoperative biochemical remission was defined on the basis of the biochemical evidence of adrenal cortical insufficiency as witnessed by the drop in the immediate postoperative serum cortisol (range, $<3 \mu \mathrm{g} / \mathrm{dL}$ ).

ЯPostoperative recurrence was defined when a patient with initial biochemical and clinical evidence for remission after operation developed symptomatic recurrence of Cushing disease with biochemical reconfirmation of pituitary-dependent endogenous hypercortisolism. Summary of patient number for each parameter:

Age: Data were available for 45 patients with no mutation and 75 patients with mutation.

BMI: Data were available for 45 patients with no mutation and 75 patients with mutation.

ACTH: Data were available for 43 patients with no mutation and 65 patients with mutation.

ACTH/Size: Data were available for 32 patients with no mutation and 51 patients with mutation.

Midnight serum cortisol: Data were available for 29 patients with no mutation and 45 patients with mutation.

Urinary free cortisol: Data were available for 28 patients with no mutation and 41 patients with mutation.

Immediate postoperative biochemical remission: Data were available for 45 patients with no mutation and 75 patients with mutation.

Postoperative recurrence: Among patients who reached immediate postoperative biochemical remission, long-term follow-up was performed in 33 cases with no mutation ( 2 were lost) and 63 cases with mutation ( 5 were lost).

Average recurrence period: In no mutation group, data were available for 8 recurrent patients, while in mutation group, data were available for 12 recurrent patients. 
Mann-Whitney $U$-test; Figure 4D). However, patients with mutated USP 8 showed comparable levels of plasma $\mathrm{ACTH}$, midnight serum cortisol and 24-h urinary free cortisol (UFC; Table 1), indicating that USP8-mutated PAs have higher ACTH production.

The rate of immediate biochemical remission after initial transsphenoidal surgery was indistinguishable between USP 8 WT and mutated patients. One month to nine years follow-up of patients receiving initial remission further demonstrated that the recurrence rate and the average recurrence period were unrelated to the USP 8 mutational status (Table 1).

\section{Discussion}

ACTH-secreting adenomas are monoclonal in nature, although sporadic in origin, and genetic alterations have been implicated in their tumorigenesis [27, 28]. However, genetic lesions have seldom been reported in these tumors. Our current study revealed that $62.04 \%$ of ACTH-secreting PAs harbor somatic mutations in exon 14 of the USP 8 gene. The number of somatic mutations per case was low, and except USP8 mutations, no other recurrent mutations were found in $12 \mathrm{ACTH}$-secreting PAs examined. Moreover, the CNV of the USP8 gene was not detected based on exome sequencing data from 12 ACTH-secreting PAs. Collectively, our study identified somatic USP8 mutations as the dominant genetic alterations in ACTH-secreting PAs, suggesting that Cushing's disease may result from genetic alterations. It is expected that other rare recurrent gene mutations may be found in Cushing's disease if we increase sample size for exome sequencing and/or examine mutational status of each of other 56 mutated genes (listed in Supplementary information, Table S2) in 108 PAs using Sanger sequencing. Importantly, none of other types of PAs harbor mutations in exon 14 of USP8. Previous studies reported that GNAS or AIP mutation-associated PAs usually secrete GH or PRL [15]. Genetic alteration of either the GNAS or AIP genes was rarely detected in ACTH-secreting PAs. Thus, our finding provides evidence that the genetic origin of PAs is heterogeneous and that ACTH-secreting PAs are pathogenically distinct. Of note, a nonsense mutation of $N R 3 C 1$, resulting in a truncated glucocorticoid receptor, was detected in one WT-USP8 patient. Inactivation of the glucocorticoid receptor signal is involved in the pathogenesis of ACTH-secreting PAs, especially for ACTH overproduction [29, 30]. Therefore, the NR3C1 mutation could be an important genetic lesion in some PAs, especially for WT-USP 8 cases.

Strikingly, all three prevalent tumor-derived USP 8 mutants fail to bind 14-3-3 proteins and display an ele- vated deubiquitinating activity toward EGFR, indicating that these represent gain-of-function mutations. Several lines of evidence support that USP 8 mutation contributes to ACTH hyperproduction: (1) USP8-mutated PAs expressed higher level of $P O M C$ mRNA, (2) patients with USP8-mutated PAs had similar levels of plasma ACTH, although PAs were markedly smaller in size, and (3) USP8 knockdown attenuated ACTH secretion in primary USP8-mutated tumor cells. Therefore, we conclude that gain-of-function USP8 mutation plays an important role in ACTH overproduction.

USP8 deubiquinates numerous proteins, and some of them are implicated in ACTH production. As a well-studied USP8 target, EGFR appears to play a central role in ACTH hyperproduction in Cushing's disease carrying USP8 mutation. We found that USP 8-mutated PAs have a higher incidence of EGFR expression, increased EGFR protein abundance and activation of downstream Erk1/2, indicating that USP8 mutations enhance EGFR signaling in tumors. Importantly, USP 8 inactivation attenuated ACTH secretion and EGFR expression in primary tumor cells. Activation of EGFR promotes POMC transcription via MAPK signaling in Cushing's disease [10]. Taken together, we conclude that USP 8 mutation sustains EGFR-MAPK signaling to promote ACTH production in Cushing's disease (Figure 5). Interestingly, low p27(Kip1) expression is more frequently observed in corticotropinoma expressing high level of EGFR [9]. Considering that activation of EGFR-MAPK signaling induces p27(Kip1) degradation, and p27(Kip1)-deficient mice develop corticotropinoma [7,31], we speculate that through activating EGFR signaling USP8 mutation accelerates p27(Kip1) degradation, representing an important molecular mechanism underlying ACTH hyperproduction (Figure 5). In addition, USP8 regulates the expression of other receptor tyrosine kinases (RTKs) such as ErbB3 and C-Met [32, 33], which share very similar signaling cascades with EGFR. Indeed, we observed the high expression level of ErbB3 and C-Met in a substantial number of USP8-mutated PAs via immunochemistry (Supplementary information, Figure S8). Smoothened, a key component of Hedgehog pathway, is deubiquitinated by USP8 [34] and activation of Hedgehog pathway induces ACTH secretion [35]. It is expected that USP 8 mutations deregulate these molecules to drive ACTH production and secretion. Further studies are required to fully understand the role of USP8 mutations in the pathogenesis of ACTH-secreting PAs and the underlying molecular mechanisms.

Our findings have important clinical implications. Most USP8-mutated PAs are small in size $(<0.5 \mathrm{~cm})$ and diffusely distributed within the sella, making them 


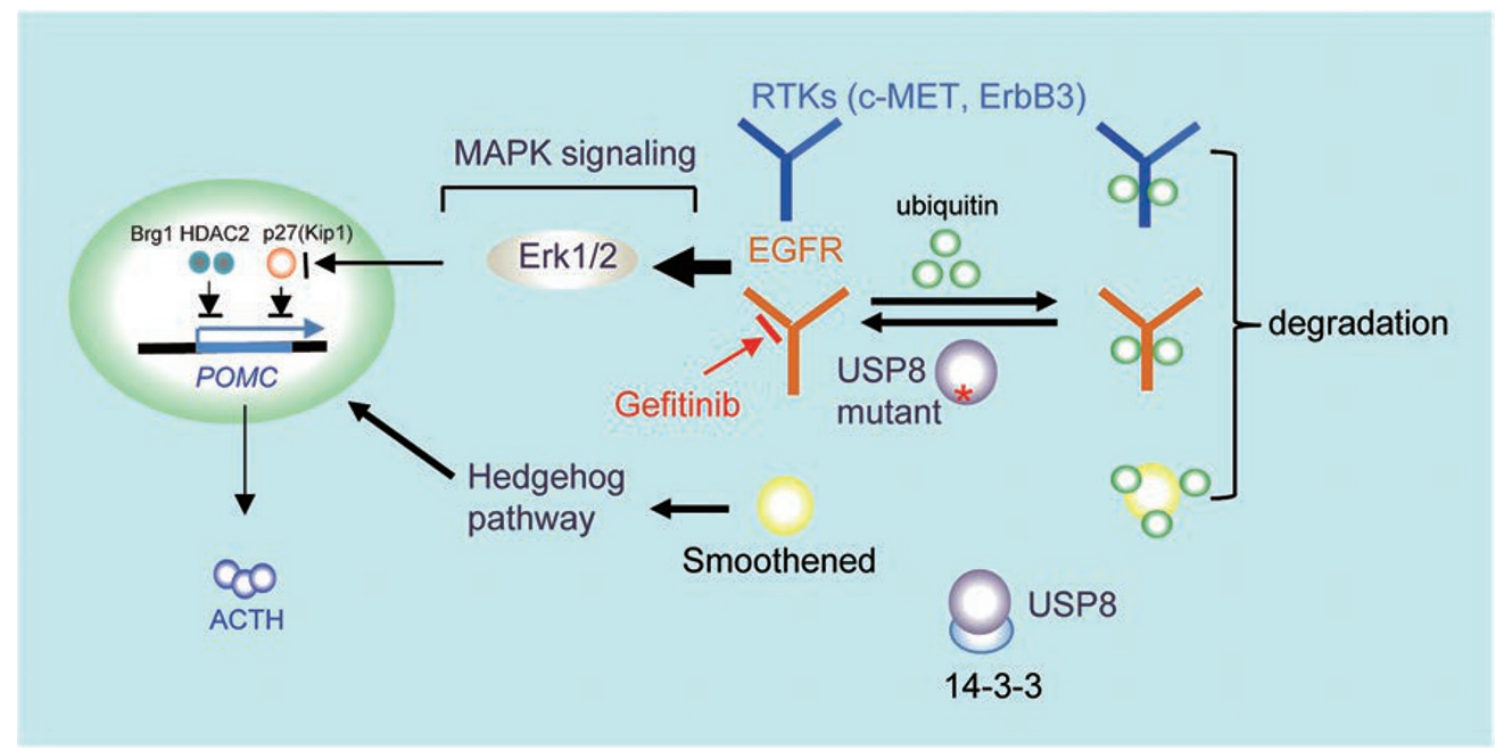

Figure 5 Mechanisms of USP8 mutation-mediated ACTH hyperproduction. The deubiquitinating enzyme USP8 can be phosphorylated allowing for association with 14-3-3 protein, which subsequently inhibits its activity. USP8 mutant fails to bind 143-3 protein, leading to an elevated USP8 activity. USP8 deubiquitinates numerous targets and protect them from degradation. EGFR is an crucial USP8 target, and deregulation of EGFR leads to increased MAPK signaling and subsequently promotes $P O M C$ transcription partially through inducing the degradation of $\mathrm{p} 27$ (Kip1), perhaps other regulators such as Brg1 and HDAC2. USP8 also regulates other RTKs such as C-MET and ErbB3, both of which potentially play a role in ACTH production. Finally, through regulating Smoothened expression and cellular location, USP8 mutation activates Hedgehog signaling, resulting in ACTH secretion. Therefore, inhibiting USP8 and/or EGFR activity represents a potential therapeutic approach for Cushing's disease.

difficult to be detected by MRI scanning and even by those experienced neurosurgeons using intraoperative neuronavigation. In contrast, almost half of the USP8WT PAs were very large and featured by parasellar invasion, increasing the difficulty and risk of surgery. In each situation, it is almost impossible to completely remove the entire tumor, which could explain the similarly low remission and high recurrence rate after initial surgery in both USP 8 WT and mutated ATCH-secreting PAs. In addition, considering that USP8-mutated PAs usually display much smaller size, we speculate that patients with small tumor even difficultly detected by MRI scanning, most likely carry USP 8 mutations. Therefore, inhibiting USP8 activity might be the first-line treatment of choice for patients with such PAs. As surgery alone seems to be unlikely to cure most cases of Cushing's disease, new therapeutic approaches are needed to specifically target tumor and suppress ACTH production $[1,5]$. Our findings strongly indicate that inhibiting USP8 catalytic activity might be a promising therapeutic strategy for Cushing's disease cases with USP 8 mutations, especially for patients with either residual or recurrent adenomas. Moreover, anti-EGFR therapy, a potential therapeutic strategy for treatment of Cushing's disease [10], seems to be more effective for patients carrying USP 8 mutation, given that these patients more likely harbor activated EGFR-MAPK signaling.

While this manuscript was in preparation, Reincke et al. [36] reported similar findings that dominant mutations in USP8 lead to Cushing's disease through activation of EGFR signaling. Of note, there are some significant differences between our study and study by Reincke et $a l$. To our knowledge, our study which includes 120 corticotrophin adenomas is the largest genetic investigation for Cushing's disease so far. The study by Reincke et al. [36] only reported 5 types of USP 8 mutations in 6 of 17 Cushing's disease cases. We reported a total of 17 types of USP8 mutations in 75 of 120 Cushing's disease cases. We further identified three highly prevalent USP $8 \mathrm{mu}-$ tations, which account for the vast majority of mutated cases $(77.61 \%)$. These data clearly define the spectrum of USP8 mutations. Furthermore, our study added valuable information which may provide guidance for the clinical practice in future: (1) Attributing to the larger sample size of the cohort, our study presents more clinical data to imply the clinical relevance of USP 8 mutation. The major novel clinical finding is that USP8-mutated corticotrophin adenomas are significantly smaller in size but 
possess higher capacity to produce ACTH $(P<0.001)$, which clearly describes the clinical characteristics of the potential subjects who may benefit from targeted therapy developed from USP8 mutations. (2) We performed inhibitory functional experiments in primary human ACTH-secreting corticotrophin adenoma cells, which was a more genetically and clinically faithful cell model. For the first time, we showed that USP8 knockdown or gefitinib treatment significantly reduced ACTH secretion in primary USP8-mutated corticotrophin adenoma cells, but not in wild-type cells. These novel findings provide direct evidence that inhibition of USP 8 and EGFR is promising for treating USP 8 -mutated corticotrophin adenoma. Taken together, the findings from our study and study by Reincke et al. will contribute to the development of novel therapies for Cushing's disease. New therapy targeting USP8 and/or EGFR might become the first-line treatment for USP8-mutated corticotrophin adenomas, especially for those surgically uncured and relapsed cases.

\section{Materials and Methods}

\section{Study patients}

Patients were recruited at the department of neurosurgery at Huashan Hospital affiliated to Shanghai Medical College, Fudan University from 2003 to 2014 for this study. A total of 120 patients (99 women and 21 men; age range from 14 to 60) with Cushing's disease were included in this study. 12 randomly selected cases were used for initial exome sequencing, and the remaining 108 cases were used to study the prevalence of USP 8 mutation (Supplementary information, Table S1). In addition, we analyzed 50 NF PAs, 50 GH-secreting PAs and 50 PRL-secreting PAs (Supplementary information, Table S1). Two normal pituitary tissues were obtained from cadaveric organ donations and tested to be suitable for immunohistochemical studies. All patients gave written informed consent, and the ethics committee at Huashan Hospital approved the study.

Cushing's disease was diagnosed using previously described and currently accepted criteria [4]. Briefly, the diagnosis of hypercortisolism was based on typical catabolic features (ie, facial plethora, purple striae, muscle atrophy of the limbs) and a combination of biochemical test results are as follows: increased awake midnight serum cortisol levels (> $7.5 \mu \mathrm{g} / \mathrm{dl}(>207.3 \mathrm{nmol}$ per liter)), elevated 24-h UFC (> $129.13 \mu \mathrm{g}(>356.9 \mathrm{nmol}))$ and nonsuppressible serum cortisol levels ( $>5 \mu \mathrm{g} / \mathrm{dl}(>138.2 \mathrm{nmol}$ per liter)) after the administration of $1 \mathrm{mg}$ of dexamethasone. The pituitary source of hypercortisolism was defined by increased morning plasma ACTH levels (> $20 \mathrm{pg} / \mathrm{ml}$ ( $>4.4$ pmol per liter)) and $>50 \%$ suppression of $24-\mathrm{h}$ UFC in the classic high-dose dexamethasone-suppression test (HDST). Finally, contrast-enhanced T1-weighted MRI scanning was used to visualize adenomas. The presence of microadenomas (usually maximal diameter $<6 \mathrm{~mm}$ ), which were undetected by MRI, was inferred by bilateral inferior petrosal sinus sampling (BIPSS). The final diagnosis of all Cushing's disease cases was histologically confirmed by two senior pa- thologists in blinded fashion. Of note, all surgically resected PAs from these patients are positive for ACTH staining, and ACTH immunostaining for 12 PAs used for whole-exome sequencing is shown in Supplementary information, Figure S9. The clinical details of 120 patients with Cushing's disease are provided in Supplementary information, Table S3. All reference ranges over the endocrine parameters in this study are provided in Supplementary information, Data S1.

\section{Whole-exome sequencing and candidate gene validation}

We performed exome sequencing of DNA from fresh tumor tissues of $12 \mathrm{ACTH}$-secreting PAs and matched blood samples on the HiSeq 2500 platform (Illumina). Exome sequencing libraries were enriched using the TruSeq ${ }^{\mathrm{TM}}$ Exome Enrichment $62 \mathrm{Mb}$ Kit (Illumina). Sanger sequencing was performed to validate the candidate gene in 108 ACTH-secreting PAs and 150 other PA types. Detailed methods of DNA extraction, library preparation and variant detection are described in Supplementary information, Data S1.

\section{DNA constructs and site-directed mutagenesis}

Full-length wild-type human USP8 and 14-3-3e expression plasmids were purchased from GE Dharmacon. cDNA encoding $14-3-3 \varepsilon$ was cloned into pGEM2 (Promega) expression vectors. The USP 8 encoding sequence was amplified and cloned into the T-easy vector (Promega), which was used for site-directed mutagenesis. The desired mutations in USP8 cDNA were introduced with the QuikChange II Site-Directed Mutagenesis Kit (Agilent Technologies). cDNA fragments encoding wild-type and mutant USP8 were subcloned into the Flag-tagged pcDH-EF-T2A-puromycin lentivirus expression vector (System Biosciences). The primers used in the mutagenesis are listed in Supplementary information, Table S4.

\section{Statistical analysis}

The statistical analysis was performed using SPSS version 16.0 (SPSS Inc.), and graphs were prepared with Prism 6.0 (GraphPad) software. Pearson's $\chi^{2}$ test and the Mann-Whitney $U$-test were used for the categorical variables and the continuous variables, respectively. $P<0.05$ was considered to be statistically significant.

Additional methods are provided in Supplementary information, Data S1.

\section{Acknowledgments}

We specially thank Genetron Health (Beijing) Co., Ltd for assistance with genomic data analysis. We gratefully acknowledge Professor Zhengping Zhuang, Dr Lei Xiong, Professor Lu Xie, Dr Baofeng Lian, Professor Xingdang Liu and Professor Zhebao Wu for helpful discussions. We thank Professor Yuqing Wang, Mrs Qiuwei Song, Ms Yun Zhang, Mrs Yun Lu and Mr Qiongji Zhu for sample collection. We also thank Jingjing Zhu and Chao Li for their technical assistance with immunohistochemical staining. This study was supported by China Pituitary Adenoma Specialist Council (CPASC), the National High Technology Research and Development Program of China (863 program; 2014AA020611), the National Program for Support of Top-Notch Young Professionals, the National Natural Science Foundation of China (81172391), Program for New Century Excellent Talents in University (NCET-10- 
0356), Shanghai Rising-Star Tracking Program (12QH1400400) to YZ; the National Basic Research program of China (973 Program; 2015CB559100), the National High Technology Research and Development Program of China (863 program; 2012AA02A515), the National Natural Science Foundation of China (31325014, 81130022 and 81272302), Shanghai Jiao Tong University Liberal Arts and Sciences Cross-Disciplinary Project (13JCRZ02), Shanghai Key Laboratory of Psychotic Disorders (13dz2260500), "Shu Guang" project supported by Shanghai Municipal Education Commission and Shanghai Education Development Foundation (12SG17) to Y-Y S.

\section{References}

1 Colao A, Boscaro M, Ferone D, Casanueva FF. Managing Cushing's disease: the state of the art. Endocrine 2014; 47:920.

2 Newell-Price J, Bertagna X, Grossman AB, Nieman LK. Cushing's syndrome. Lancet 2006; 367:1605-1617.

3 Melmed S. The Pituitary. 3rd ed. Amsterdam: Elsevier 2011:712-713.

4 Nieman LK, Biller BM, Findling JW, et al. The diagnosis of Cushing's syndrome: an Endocrine Society Clinical Practice Guideline. J Clin Endocrinol Metab 2008; 93:1526-1540.

5 Biller BM, Grossman AB, Stewart PM, et al. Treatment of adrenocorticotropin-dependent Cushing's syndrome: a consensus statement. J Clin Endocrinol Metab 2008; 93:2454-2462.

6 Bilodeau S, Vallette-Kasic S, Gauthier Y, et al. Role of Brg1 and HDAC2 in GR trans-repression of the pituitary POMC gene and misexpression in Cushing disease. Genes Dev 2006; 20:2871-2886.

7 Lidhar K, Korbonits M, Jordan S, et al. Low expression of the cell cycle inhibitor p27Kip1 in normal corticotroph cells, corticotroph tumors, and malignant pituitary tumors. $J$ Clin Endocrinol Metab 1999; 84:3823-3830.

8 Kiyokawa H, Kineman RD, Manova-Todorova KO, et al. Enhanced growth of mice lacking the cyclin-dependent kinase inhibitor function of p27(Kip1). Cell 1996; 85:721-732.

9 Theodoropoulou M, Arzberger T, Gruebler Y, et al. Expression of epidermal growth factor receptor in neoplastic pituitary cells: evidence for a role in corticotropinoma cells. $J$ Endocrinol 2004; 183:385-394.

10 Fukuoka H, Cooper O, Ben-Shlomo A, et al. EGFR as a therapeutic target for human, canine, and mouse ACTH-secreting pituitary adenomas. J Clin Invest 2011; 121:4712-4721.

11 Vallar L, Spada A, Giannattasio G. Altered Gs and adenylate cyclase activity in human GH-secreting pituitary adenomas. Nature 1987; 330:566-568.

12 Weinstein LS, Shenker A, Gejman PV, Merino MJ, Friedman E, Spiegel AM. Activating mutations of the stimulatory $\mathrm{G}$ protein in the McCune-Albright syndrome. $N$ Engl J Med 1991; 325:1688-1695.

13 Chahal HS, Stals K, Unterlander M, et al. AIP mutation in pituitary adenomas in the 18 th century and today. $N$ Engl J Med 2011; 364:43-50.

14 Cazabat L, Bouligand J, Salenave S, et al. Germline AIP mutations in apparently sporadic pituitary adenomas: prevalence in a prospective single-center cohort of 443 patients. $J$ Clin Endocrinol Metab 2012; 97:E663-E670.
15 Stratakis CA, Tichomirowa MA, Boikos S, et al. The role of germline AIP, MEN1, PRKAR1A, CDKN1B and CDKN2C mutations in causing pituitary adenomas in a large cohort of children, adolescents, and patients with genetic syndromes. Clin Genet 2010; 78:457-463.

16 Kawashima ST, Usui T, Sano T, et al. P53 gene mutation in an atypical corticotroph adenoma with Cushing's disease. Clin Endocrinol (Oxf) 2009; 70:656-657.

17 De Menis E, Roncaroli F, Calvari V, et al. Corticotroph adenoma of the pituitary in a patient with X-linked adrenal hypoplasia congenita due to a novel mutation of the $D A X-1$ gene. Eur J Endocrinol 2005; 153:211-215.

18 Sahakitrungruang T, Srichomthong C, Pornkunwilai S, et al. Germline and somatic DICER1 mutations in a pituitary blastoma causing infantile-onset Cushing's disease. J Clin Endocrinol Metab 2014; 99:E1487-1492.

19 Karl M, Lamberts SW, Koper JW, et al. Cushing's disease preceded by generalized glucocorticoid resistance: clinical consequences of a novel, dominant-negative glucocorticoid receptor mutation. Proc Assoc Am Physicians 1996; 108:296307.

20 Naviglio S, Mattecucci C, Matoskova B, et al. UBPY: a growth-regulated human ubiquitin isopeptidase. EMBO J 1998; 17:3241-3250.

21 Mizuno E, Iura T, Mukai A, Yoshimori T, Kitamura N, Komada M. Regulation of epidermal growth factor receptor down-regulation by UBPY-mediated deubiquitination at endosomes. Mol Biol Cell 2005; 16:5163-5174.

22 Mizuno E, Kitamura N, Komada M. 14-3-3-dependent inhibition of the deubiquitinating activity of UBPY and its cancellation in the M phase. Exp Cell Res 2007; 313:3624-3634.

23 Berlin I, Schwartz H, Nash PD. Regulation of epidermal growth factor receptor ubiquitination and trafficking by the USP8.STAM complex. J Biol Chem 2010; 285:34909-34921.

24 Yaffe MB, Rittinger K, Volinia S, et al. The structural basis for 14-3-3:phosphopeptide binding specificity. Cell 1997; 91:961-971.

25 Wilson CB. A decade of pituitary microsurgery. The Herbert Olivecrona lecture. J Neurosurg 1984; 61:814-833.

26 Knosp E, Steiner E, Kitz K, Matula C. Pituitary adenomas with invasion of the cavernous sinus space: a magnetic resonance imaging classification compared with surgical findings. Neurosurgery 1993; 33:610-617; discussion 617-618.

27 Herman V, Fagin J, Gonsky R, Kovacs K, Melmed S. Clonal origin of pituitary adenomas. J Clin Endocrinol Metab 1990; 71:1427-1433.

28 Biller BM, Alexander JM, Zervas NT, Hedley-Whyte ET, Arnold A, Klibanski A. Clonal origins of adrenocorticotropin-secreting pituitary tissue in Cushing's disease. J Clin Endocrinol Metab 1992; 75:1303-1309.

29 Drouin J, Bilodeau S, Vallette S. Of old and new diseases: genetics of pituitary ACTH excess (Cushing) and deficiency. Clin Genet 2007; 72:175-182.

30 Chrousos GP, Detera-Wadleigh SD, Karl M. Syndromes of glucocorticoid resistance. Ann Intern Med 1993; 119:11131124.

31 Chu IM, Hengst L, Slingerland JM. The Cdk inhibitor p27 in human cancer: prognostic potential and relevance to anticancer therapy. Nat Rev Cancer 2008; 8:253-267. 
32 Niendorf S, Oksche A, Kisser A, et al. Essential role of ubiquitin-specific protease 8 for receptor tyrosine kinase stability and endocytic trafficking in vivo. Mol Cell Biol 2007; 27:5029-5039.

33 Byun S, Lee SY, Lee J, et al. USP8 is a novel target for overcoming gefitinib resistance in lung cancer. Clin Cancer Res 2013; 19:3894-3904.

34 Xia R, Jia H, Fan J, Liu Y, Jia J. USP8 promotes smoothened signaling by preventing its ubiquitination and changing its subcellular localization. PLoS Biol 2012; 10:e1001238.

35 Vila G, Theodoropoulou M, Stalla J, et al. Expression and function of sonic hedgehog pathway components in pituitary adenomas: evidence for a direct role in hormone secretion and cell proliferation. J Clin Endocrinol Metab 2005; 90:66876694.

36 Reincke M, Sbiera S, Hayakawa A, et al. Mutations in the deubiquitinase gene USP8 cause Cushing's disease. Nat Genet 2015; 47:31-38.

(Supplementary information is linked to the online version of the paper on the Cell Research website.)

(c) (i) () $\odot$ This work is licensed under the Creative Commons Attribution-NonCommercial-No Derivative Works 3.0 Unported License. To view a copy of this license, visit http:// creativecommons.org/licenses/by-nc-nd/3.0 